\title{
Small deformations of topological algebras
}

\author{
by \\ Mati Abel (Tartu) and Krzysztof Jarosz (Edwardsville, IL)
}

\begin{abstract}
We investigate stability of various classes of topological algebras and individual algebras under small deformations of multiplication.
\end{abstract}

1. Introduction. By an $\varepsilon$-deformation (or perturbation) of a Banach algebra $(A, \cdot)$ we mean a second multiplication $\times$ defined on the same Banach space $A$ such that the norm of the bilinear map $\times-\cdot$ is not greater than $\varepsilon$, that is,

$$
\|a \times b-a \cdot b\| \leq \varepsilon\|a\|\|b\| \quad \text { for all } a, b \in A .
$$

We always assume that a multiplication is associative, but not necessarily commutative.

Small deformations of Banach algebras have been investigated since the early seventies by R. Rochberg [15-21], B. E. Johnson [13, 14], K. Jarosz [5-12], and others. While some of the results are applicable to general Banach algebras, the main interest has been in the deformations of uniform algebras in connection with small deformations of holomorphic structures. There are three basic problems in the field:

(1) To characterize stable Banach algebras. A Banach algebra is called stable if there is an $\varepsilon_{0}>0$ such that for any $\varepsilon$-deformation $\times$ with $\varepsilon<\varepsilon_{0}$, the algebras $(A, \cdot)$ and $(A, \times)$ are isomorphic.

(2) To characterize stable properties. We say that a property $P$ is stable if there exists an $\varepsilon_{0}>0$ such that for any algebra $(A, \cdot)$ having property $P$ and any $\varepsilon$-deformation $\times$ of that algebra with $\varepsilon<\varepsilon_{0}$, the algebra $(A, \times)$ also has property $P$.

(3) To characterize continuous or differentiable structures on the space of all small deformations.

2000 Mathematics Subject Classification: Primary 46H05, 46H40; Secondary 46J05, $46 \mathrm{~A} 03$.

Key words and phrases: topological algebras, small perturbations, small deformations, Banach algebras, stability.

Research partially supported by a grant from the National Research Council. 
For example the Banach algebras $C(X), A(\mathbb{D}), H^{\infty}(\mathbb{D})$, and the properties "Dirichlet" or " $\partial A=\mathrm{Ch} A$ " are stable $[5,8,13,20]$, while the algebras of analytic functions of one variable defined on nonsimply connected domains with nonempty interiors are not stable [21].

The concept of small deformations of algebras provides a very natural definition of a small deformation of a Riemann manifold $\Omega$ : we call $\Omega^{\prime}$ a small deformation of $\Omega$ if an algebra $A_{\Omega^{\prime}}$ of analytic functions on $\Omega^{\prime}$ is isomorphic to a small deformation $\left(A_{\Omega}, \times\right)$ of an analogous algebra $A_{\Omega}$. It turns out that for one-dimensional Riemann manifolds, this approach, with $A_{\Omega}$ equal to the algebra of functions analytic on $\Omega$ and continuous on $\bar{\Omega}$, is exactly equivalent to the theory of quasiconformal deformations [21]. On the other hand, almost nothing is known about small deformations of algebras of analytic functions of several variables [10]. The problem is of particular importance since an answer may provide a multidimensional quantitative version of the Riemann Mapping Theorem.

In this paper we extend the theory of small deformations to topological algebras. There are several ways to generalize the definition of a small deformation to the class of algebras equipped with a topology but without a norm. In the sections following the Definitions and notation we discuss briefly two very natural extensions; we show, however, that both have serious limitations. In the main section (Section 4), we arrive at what we believe is the right definition, extending the concept of stability and small deformations to the class of topological algebras. We show that in many cases the theory is analogous to the theory of deformations of Banach algebras, while it may provide an even better framework for working with small deformations of holomorphic manifolds. The paper does not provide a comprehensive theory of deformations of topological algebras; it is rather intended as an invitation to this mostly open area of research.

By a topological algebra we mean here a topological vector space with an associative and jointly continuous multiplication. In general such an algebra may be quite pathological, e.g. all elements other than multiples of the identity may have unbounded spectrum, the multiplicative inversion $a \mapsto a^{-1}$ may be discontinuous, the set of invertible elements may be nonopen, etc. In this paper we are particularly interested in small deformations of reasonably nice topological algebras, like topological function algebras, $m$-convex algebras, and $Q$-algebras. We will also often assume that the algebras under consideration are complete, because several examples will show that without this assumption, small deformations even of a normed algebra can be pathological.

2. Definitions and notation. Since the terminology concerning topological algebras varies, we state for the record the definitions of some of the classes and properties of algebras we will refer to. 
Definition 1. By a Fréchet algebra we mean a complete metrizable topological algebra.

Definition 2. By a Gelfand-Mazur algebra we mean a topological algebra $A$ such that for each closed two-sided regular ideal $M$ which is maximal as a left or as a right ideal, the quotient algebra $A / M$ is topologically isomorphic to the scalar field.

Definition 3. By an $m$-convex algebra we mean an algebra whose topology can be given by a family $\left\{p_{\lambda}: \lambda \in \Lambda\right\}$ of $m$-convex seminorms, that is, seminorms satisfying

$$
p_{\lambda}(a b) \leq p_{\lambda}(a) p_{\lambda}(b) \quad \text { for all } a, b \in A, \lambda \in \Lambda .
$$

If $A$ is both a Fréchet algebra and $m$-convex, then its topology can be given by an increasing sequence of $m$-convex seminorms.

DEFINITION 4. By a topological function algebra we mean a closed subalgebra $A$ of an algebra $C(X)$ of all continuous functions defined on a completely regular Hausdorff space $X$; we assume that the topology of $A$ is that of uniform convergence on compact subsets of $X$.

The algebra $C(X)$ is metrizable if and only if $X$ is hemicompact; it is complete if and only if $X$ is a $k_{R^{-}}$-space $([1, \mathrm{pp} .63-65])$. Since every locally compact and $\sigma$-compact Hausdorff space $X$ is hemicompact and a $k_{R^{-}}$-space, for such spaces $X$, the topological algebra $C(X)$ is lmc and Fréchet.

Definition 5. By a $Q$-algebra we mean a topological algebra $A$ such that the group of its quasi-invertible elements is open.

If an algebra $A$ has a unit $e$ then $a \in A$ is called quasi-invertible if $e-a$ is invertible. If an algebra does not have a unit we call an element $a$ quasi-invertible if $e-a$ is invertible in the algebra $A \oplus\{\lambda e: \lambda \in \mathbb{C}\}$ obtained from $A$ by adding a unit element $e$. Equivalently, $a$ is quasi-invertible if there is an element $a^{0}$, called a quasi-inverse of $a$, such that $a+a^{0}=a a^{0}$; in the algebra $A \oplus\{\lambda e: \lambda \in \mathbb{C}\}$, the inverse of $e-a$ is $e-a^{0}$.

In spite of the fact that $Q$-algebras may be noncomplete they share many of the fundamental properties of Banach algebras; in fact, several of these properties characterize the $Q$-property. $Q$-algebras, like for example $C^{\infty}[0,1]$, algebras of rapidly decreasing functions, algebras of functions with compact support, and others, play a crucial role in the theory of distributions, pseudodifferential operators, etc. [3].

Definition 6. An element $a$ of a topological algebra $A$ is called bounded if for some nonzero complex number $\lambda_{a}$ the set $\left\{\left(a / \lambda_{a}\right)^{n}: n \in \mathbb{N}\right\}$ is bounded in $A$. A topological algebra in which all elements are bounded is called a topological algebra with bounded elements. 
If the topology of an algebra is given by a family of seminorms we also have a related uniform property.

Definition 7. Let $A$ be a topological algebra with the topology given by a fixed family $\left\{p_{j}: j \in J\right\}$ of seminorms. We say that $A$ is an algebra with uniformly bounded elements if for any $a \in A$ there is a positive number $\lambda_{a}$ such that

$$
\sup _{j \in J} \sup _{n} p_{j}\left(\left(a / \lambda_{a}\right)^{n}\right)<\infty .
$$

An example of a topological algebra with uniformly bounded elements whose topology cannot be given by a norm is the algebra $C_{\mathrm{b}}(\mathbb{R})$ of all continuous bounded functions on the real line with the seminorms

$$
p_{n}(f)=\sup _{-n \leq t \leq n}|f(t)|, \quad f \in C_{\mathrm{b}}(\mathbb{R}), n \in \mathbb{N} .
$$

3. Strong stability and weak stability. While the original definition (1.1) of a small deformation of a Banach algebra is expressed by a formula involving the norm of the algebra, the concept of stability of the algebra, or stability of a particular property, can be described in topological terms.

DEFINITION 8. We call a topological algebra $(A, \cdot)$ strongly stable if there is a neighborhood $V$ of $\cdot \in L_{2}(A)$ such that for each associative map $\times \in V$ the algebras $(A, \cdot)$ and $(A, \times)$ are topologically isomorphic.

Here, by $L_{2}(A)$ we denote the space of all continuous bilinear maps $T: A \times A \rightarrow A$ equipped with the bounded-open topology, that is, the topology with a base of neighborhoods of zero defined by

$$
\left\{T \in L_{2}(A): T\left(B^{2}\right) \subset U\right\},
$$

where $B$ is a bounded subset of $A$ and $U$ a neighborhood of zero in $A$.

The above definition coincides with the classical one for Banach algebras where the bounded-open topology is just the norm operator topology. So it may seem to be the most natural generalization of stability from Banach algebras to topological algebras. However, in a typical topological algebra, bounded sets are quite small and rarely have nonempty interior. Consequently, the requirement in the definition that all associative maps $\times$ from $V$ produce an isomorphic algebra is very strong. So strong, indeed, that even very nice and rigid looking topological non-Banach algebras are not strongly stable. The following theorem which we provide without a proof lists several algebras that are not strongly stable.

THEOREM 1. The following topological algebras are not strongly stable:

- the algebra $C(\mathbb{C})$ of all continuous functions defined on the complex plane $\mathbb{C}$,

- the algebra $\operatorname{Hol}(\mathbb{C})$ of all holomorphic functions on $\mathbb{C}$, 
- the algebra $\operatorname{Hol}(\mathbb{D})$ of all holomorphic functions defined on the open unit disc $\mathbb{D}$,

- the algebra $C^{\infty}([0,1])$ of all infinitely differentiable functions on the unit interval,

- the algebra $A^{\infty}(\overline{\mathbb{D}})$ of all $C^{\infty}$ functions defined on the closed unit disc $\overline{\mathbb{D}}$ which are holomorphic on the interior of the disc.

In the first three cases we equip the algebra with the topology of uniform convergence on compact sets and define multiplication pointwise. In the last two cases we equip $A$ with the topology of uniform convergence of all the derivatives.

The algebras above are all functionally semisimple, metrizable, complete, and $m$-convex. In fact, such algebras often serve as standard examples of the simplest topological algebras outside the class of Banach algebras. One can show that in any neighborhood of the original multiplication of such an algebra there are multiplications with very different properties, for example multiplications with nontrivial radical.

The definition of strong stability was too strong since it did not guarantee that the new multiplication is uniformly close to the original one. If the topology of an algebra $A$ can be defined by a family $\left\{p_{\lambda}: \lambda \in \Lambda\right\}$ of $m$ convex seminorms, one can define a class of deformations that are uniformly close to the original one and define the corresponding stability property.

Definition 9. Let $(A, \cdot)$ be an $m$-convex algebra with a unit $e$, and let $\left\{p_{\lambda}: \lambda \in \Lambda\right\}$ be the family of all $m$-convex continuous seminorms on $A$. We call $(A, \cdot)$ weakly stable if there is an $\varepsilon>0$ such that for any associative multiplication $\times$ on $A$ with

$$
p_{\lambda}(a \cdot b-a \times b) \leq \varepsilon p_{\lambda}(a) p_{\lambda}(b) \quad \text { for all } a, b \in A, \lambda \in \Lambda,
$$

the algebras $(A, \cdot)$ and $(A, \times)$ are topologically isomorphic.

Notice that the new algebra $(A, \times)$ is also $m$-convex: indeed, from (3.1) we get

$$
p_{\lambda}(a \times b) \leq(1+\varepsilon) p_{\lambda}(a) p_{\lambda}(b) \quad \text { for } a, b \in A, \lambda \in \Lambda
$$

so

$$
q_{\lambda}:=(1+\varepsilon) p_{\lambda}
$$

are $m$-convex seminorms on $(A, \times)$.

Again, the definition is very natural; however, this time most complete topological algebras are weakly stable - indeed, we have the following theorem which we again present without proof.

THEOREM 2. Any unital semisimple complete commutative $m$-convex Hausdorff algebra is weakly stable.

At the same time, noncomplete algebras may still behave pathologically. 
ExAMPLE 1. Let $A$ be the algebra of all polynomials of a variable $t$ with pointwise multiplication and the sup norm on the unit interval:

$$
\|p\|=\sup \{|p(t)|: 0 \leq t \leq 1\} \quad \text { for } p \in A .
$$

Fix $\varepsilon>0$ and define a new multiplication $\times$ on $A$ by

$$
(p \times q)(t)=(1+\varepsilon t) p(t) q(t) \quad \text { for } p, q \in A .
$$

It is easy to check that the new multiplication satisfies (3.1); however, the algebras $A$ and $(A, \times)$ are not isomorphic, since $A$ has a unit while $(A, \times)$ does not.

4. Stability. Since the last two definitions were not fully satisfactory, providing too weak or too strong a property, let us examine again the original definition of a small deformation of a Banach algebra. The definition involves not only the topology but a specific norm of the algebra. If we replace the original norm $\|\cdot\|$ on $A$ by an equivalent norm $p(\cdot)$, then (1.1) becomes

$$
p(a \times b-a \cdot b) \leq \varepsilon C p(a) p(b) \quad \text { for all } a, b \in A,
$$

where the constant $C$ can be arbitrarily large or arbitrarily small. Hence small deformations of a Banach algebra are related not only to the particular algebraic and topological structures on $A$, but also to a specific norm on that space, rather than the family of all equivalent norms. Consequently, it will only be natural to define small perturbations not for an abstract topological algebra, but for a concrete topological algebra equipped with a given set of seminorms.

Definition 10. Let $(A, \cdot)$ be an algebra and $\Xi=\left\{p_{\lambda}: \lambda \in \Lambda\right\}$ a family of $m$-convex seminorms. Assume that the set $\Xi$ separates the points of $A$, so that $(A, \cdot, \Xi)$ is an $m$-convex Hausdorff algebra. We call $(A, \cdot, \Xi)$ stable if there is an $\varepsilon>0$ such that for any associative multiplication $\times$ on $A$ with

$$
p(a \cdot b-a \times b) \leq \varepsilon p(a) p(b) \quad \text { for } a, b \in A \text { and } p \in \Xi,
$$

the algebras $(A, \cdot)$ and $(A, \times)$ are topologically isomorphic.

We call a property $P$ stable (or stable in a given class of topological algebras) if for any algebra $(A, \cdot, \Xi)$ with property $P$ there is an $\varepsilon>0$ such that for any $\varepsilon$-deformation $\times$ of that algebra defined as above, the new algebra $(A, \times, \Xi)$ also has property $P$.

We notice that, as in the case of weak stability, the new algebra $(A, \times)$ is also $m$-convex, with $m$-convex seminorms defined by

$$
q_{\lambda}:=(1+\varepsilon) p_{\lambda} \quad \text { for } \lambda \in \Lambda \text {. }
$$

4.1. Basic stable properties. The next proposition shows that, for a large class of topological algebras, a small perturbation of an algebra with a unit is again a unital algebra. 
Proposition 1. For the sequentially complete m-convex algebras the property of having a unit is stable.

Proof. Assume $A$ is a unital sequentially complete $m$-convex algebra; we denote by $e$ the unit of $A$. Let $\times$ be another multiplication on $A$ that satisfies (4.1) with an $\varepsilon<1$. Put

$$
M: A \rightarrow A, \quad M(a)=e \times a,
$$

and

$$
T=\sum_{n=0}^{\infty}(\mathrm{Id}-M)^{n}: A \rightarrow A,
$$

where Id is the identity map on $A$.

For any $m$-convex seminorm $p$ we may assume that $p(e)=1$ (see [23]) and we have

$$
\begin{aligned}
p((\operatorname{Id}-M)(a)) & =p(a-e \times a) \leq \varepsilon p(a), \\
p\left((\operatorname{Id}-M)^{2}(a)\right) & =p((\operatorname{Id}-M)(a)-e \times(\operatorname{Id}-M)(a)) \\
& \leq \varepsilon p((\operatorname{Id}-M)(a)) \leq \varepsilon^{2} p(a), \ldots, \\
p\left((\operatorname{Id}-M)^{n}(a)\right) & \leq \varepsilon^{n} p(a) \quad \text { for all } n .
\end{aligned}
$$

So, since $A$ is sequentially complete, the series defining $T$ is convergent. We have

$$
\begin{aligned}
M \circ T & =T \circ M=\sum_{n=0}^{\infty}(\mathrm{Id}-M)^{n} \circ M=\sum_{n=0}^{\infty}(\mathrm{Id}-M)^{n} \circ(\mathrm{Id}-(\mathrm{Id}-M)) \\
& =\sum_{n=0}^{\infty}(\mathrm{Id}-M)^{n}-\sum_{n=1}^{\infty}(\mathrm{Id}-M)^{n}=\mathrm{Id},
\end{aligned}
$$

so $T=M^{-1}$, in particular $M$ is surjective.

For $e_{0}:=M^{-1}(e)$ we get $e \times e_{0}=M\left(e_{0}\right)=e$. Hence, for any $b$ in $A$,

$$
M\left(e_{0} \times b-b\right)=e \times e_{0} \times b-e \times b=e \times b-e \times b=0,
$$

and since $M$ is injective it follows that $e_{0} \times b-b=0$ for all $b \in A$, so $e_{0}$ is a unit of $(A, \times)$.

REMARK 1. Based on the last proposition we may always assume that for a sequentially complete $m$-convex algebra a new multiplication has the same unit as the original one. To justify this we need to show that there is a third multiplication $*$ on $A$ such that the algebras $(A, \times)$ and $(A, *)$ are topologically isomorphic, $*$ is a small deformation of $(A, \cdot)$, that is, there is an $\varepsilon^{\prime}=O(\varepsilon)$ such that

$$
p(a \cdot b-a * b) \leq \varepsilon^{\prime} p(a) p(b) \quad \text { for } a, b \in A \text { and } p \in \Xi,
$$

and the algebras $(A, \cdot)$ and $(A, *)$ have the same unit. 
To this end assume that $\times$ is a new multiplication on $A$ satisfying (4.1) with $\varepsilon<1$, denote by $e$ the unit of the original multiplication on $A$, and by $e_{0}$ the unit of the $\times$-multiplication. Put

$$
\Phi: A \rightarrow A, \quad \Phi(a)=a \cdot e_{0} .
$$

Arguing exactly as for the map $M$ in the proof of the last proposition one can check that $\Phi$ is an invertible map from $A$ onto itself with

Moreover, as before,

$$
\Phi^{-1}=\sum_{n=0}^{\infty}(\mathrm{Id}-\Phi)^{n} .
$$

$$
\begin{aligned}
p_{\lambda}\left(\Phi^{-1}(a)\right) & =p_{\lambda}\left(\sum_{n=0}^{\infty}(\operatorname{Id}-\Phi)^{n}(a)\right) \\
& \leq \sum_{n=0}^{\infty} \varepsilon^{n} p_{\lambda}(a)=\frac{p_{\lambda}(a)}{1-\varepsilon} \quad \text { for all } a \in A \text { and } \lambda \in \Lambda
\end{aligned}
$$

We define a third multiplication $*$ on $A$ by

$$
a * b=\Phi^{-1}(\Phi(a) \times \Phi(b)) \quad \text { for all } a, b \in A .
$$

Notice that:

- $\Phi$ is an algebra isomorphism from $(A, *)$ onto $(A, \times)$, so the algebras $(A, *)$ and $(A, \times)$ are isomorphic.

- Since

$$
e_{0}=\Phi^{-1}(e)=\sum_{n=0}^{\infty}(\operatorname{Id}-\Phi)^{n}(e),
$$

from (4.3) we get

$$
p_{\lambda}\left(e_{0}\right) \leq \frac{1}{1-\varepsilon}, \quad p_{\lambda}\left(e-e_{0}\right) \leq \frac{\varepsilon}{1-\varepsilon} \quad \text { for all } \lambda \in \Lambda,
$$

so, by (4.3) it follows that for all $a, b \in A$ and $\lambda \in \Lambda$ we have

$$
\begin{aligned}
p_{\lambda}(a & \cdot b-a * b)=p_{\lambda}\left(a \cdot b-\Phi^{-1}\left(\left(a \cdot e_{0}\right) \times\left(b \cdot e_{0}\right)\right)\right) \\
& \leq \frac{1}{1-\varepsilon} p_{\lambda}\left(\Phi\left(a \cdot b-\left(a \cdot e_{0}\right) \times\left(b \cdot e_{0}\right)\right)\right) \\
& =\frac{1}{1-\varepsilon} p_{\lambda}\left(a \cdot b \cdot e_{0}-\left(a \cdot e_{0}\right) \times\left(b \cdot e_{0}\right)\right) \\
& =\frac{1}{1-\varepsilon} p_{\lambda}\left(a \cdot\left(b \cdot e_{0}\right)-a \times\left(b \cdot e_{0}\right)+a \times\left(b \cdot e_{0}\right)-\left(a \cdot e_{0}\right) \times\left(b \cdot e_{0}\right)\right) \\
& \leq \frac{1}{1-\varepsilon}\left[\varepsilon p_{\lambda}(a) p_{\lambda}\left(b \cdot e_{0}\right)+p_{\lambda}\left(\left(a-a \cdot e_{0}\right) \times\left(b \cdot e_{0}\right)\right)\right] \\
& \leq \frac{1}{1-\varepsilon}\left[\varepsilon p_{\lambda}(a)\left(p_{\lambda}(b) p_{\lambda}\left(e_{0}\right)+(1+\varepsilon) p_{\lambda}(a) p_{\lambda}\left(e-e_{0}\right) p_{\lambda}(b) p_{\lambda}\left(e_{0}\right)\right)\right] \\
& \leq \varepsilon^{\prime} p_{\lambda}(a) p_{\lambda}(b)
\end{aligned}
$$


with

$\varepsilon^{\prime}:=\frac{1}{1-\varepsilon} p_{\lambda}\left(e_{0}\right)\left[\varepsilon+(1+\varepsilon) p_{\lambda}\left(e-e_{0}\right)\right] \leq \frac{1}{(1-\varepsilon)^{2}}\left(\varepsilon+\frac{(1+\varepsilon) \varepsilon}{1-\varepsilon}\right)=\frac{2 \varepsilon}{(1-\varepsilon)^{3}}$.

Hence $*$ is an $\varepsilon^{\prime}$-deformation of $(A, \cdot)$.

- The algebras $(A, \cdot)$ and $(A, *)$ have the same unit $e$.

Proposition 2. Assume $(A, \cdot, \Xi)$ is a topological algebra with the topology given by the family $\Xi=\left\{p_{\lambda}: \lambda \in \Lambda\right\}$ of $m$-convex seminorms, and let $\times$ be an $\varepsilon$-deformation of $(A, \cdot, \Xi)$. Then $\operatorname{ker} p_{\lambda}, \lambda \in \Lambda$, are closed twosided ideals in both algebras $(A, \cdot, \Xi)$ and $(A, \times, \Xi)$, and for any $\lambda \in \Lambda$ and $a_{1}, a_{2}, b_{1}, b_{2} \in A$ we have

$$
p_{\lambda}\left(a_{1}-a_{2}\right)=0=p_{\lambda}\left(b_{1}-b_{2}\right) \Rightarrow p_{\lambda}\left(a_{1} \times b_{1}-a_{2} \times b_{2}\right)=0 .
$$

Proof. Fix $\lambda \in \Lambda$ and assume that $a_{1}, a_{2}, b_{1}, b_{2} \in A$ are such that

$$
p_{\lambda}\left(a_{1}-a_{2}\right)=0=p_{\lambda}\left(b_{1}-b_{2}\right) \text {. }
$$

We have

$$
\begin{aligned}
p_{\lambda}\left(\left(a_{1}-a_{2}\right) \times b_{1}\right) & \leq p_{\lambda}\left(\left(a_{1}-a_{2}\right) \times b_{1}-\left(a_{1}-a_{2}\right) b_{1}\right)+p_{\lambda}\left(a_{1}-a_{2}\right) p_{\lambda}\left(b_{1}\right) \\
& \leq \varepsilon p_{\lambda}\left(a_{1}-a_{2}\right) p_{\lambda}\left(b_{1}\right)=0,
\end{aligned}
$$

So

$$
p_{\lambda}\left(a_{1} \times b_{1}-a_{2} \times b_{1}\right)=0 .
$$

By symmetry we also have

$$
p_{\lambda}\left(a_{2} \times b_{1}-a_{2} \times b_{2}\right)=0 .
$$

Hence

$$
p_{\lambda}\left(a_{1} \times b_{1}-a_{2} \times b_{2}\right)=0 .
$$

REMARK 2. Assume $(A, \cdot, \Xi)$ is a topological algebra with the topology given by the family $\Xi=\left\{p_{\lambda}: \lambda \in \Lambda\right\}$ of $m$-convex seminorms, let $\times$ be an $\varepsilon$-deformation of $(A, \cdot, \Xi)$, and let $\bar{A}_{\lambda}$ be the completion of the quotient algebra $A / \operatorname{ker} p_{\lambda}$. Based on the above proposition we can define two multiplications on each of the Banach spaces $\bar{A}_{\lambda}: \cdot_{\lambda}$ induced by the original multiplication, and $\times_{\lambda}$ induced by $\times$. We have

$$
\left\|f \cdot{ }_{\lambda} g-f \times_{\lambda} g\right\|_{\lambda} \leq \varepsilon\|f\|_{\lambda}\|g\|_{\lambda} \quad \text { for all } f, g \in \bar{A}_{\lambda},
$$

where $\|\cdot\|_{\lambda}$ is the quotient norm. Hence $\left(\bar{A}_{\lambda}, \times_{\lambda}\right)$ is an $\varepsilon$-deformation of the Banach algebra $\left(\bar{A}_{\lambda}, \cdot{ }_{\lambda}\right)$.

4.2. Stable algebras. The next theorem states that the simplest topological function algebras, that is, the algebras of all continuous functions, are stable.

TheOREM 3. Let $A=C(X)$ be the algebra of all continuous functions defined on a completely regular Hausdorff $k$-space $X$ with the usual pointwise 
multiplication, and let $\mathcal{K}$ be a cover of $X$ consisting of compact sets. Put

$$
\|f\|_{K}=\sup \{|f(z)|: z \in K\} \quad \text { for } f \in A, K \in \mathcal{K} .
$$

Then the algebra $\left(A,\left\{\|\cdot\|_{K}: K \in \mathcal{K}\right\}\right)$ is stable.

Proof. Assume $\times$ is another multiplication on $C(X)$ such that

$$
\|f g-f \times g\|_{K} \leq \varepsilon\|f\|_{K}\|g\|_{K} \quad \text { for all } f, g \in C(X), K \in \mathcal{K} .
$$

It follows that

$$
\|f g-f \times g\|_{X} \leq \varepsilon\|f\|_{X}\|g\|_{X} \quad \text { for all } f, g \in C_{\mathrm{b}}(X),
$$

where $\left(C_{\mathrm{b}}(X),\|\cdot\|_{X}\right)$ is the Banach algebra of all bounded continuous functions on $X$ equipped with the sup norm. Since $\left(C_{\mathrm{b}}(X),\|\cdot\|_{X}\right)$ is isometrically isomorphic with the stable Banach algebra $C(\beta X)$ of all continuous functions on the Cech-Stone compactification $\beta X$ of $X$, there is a map ([5, Ex. 17.3])

$$
\Psi: C(\beta X) \rightarrow C(\beta X)
$$

with $\|\Psi\| \leq c \varepsilon$, where $c$ is an absolute constant, such that

$$
T:=\mathrm{Id}+\Psi
$$

is an algebra isomorphism from $(C(\beta X), \times)$ onto $C(\beta X)$, that is,

$$
T(f \times g)=T(f) T(g) \quad \text { for } f, g \in C_{\mathrm{b}}(X) .
$$

Hence for any $x \in X$ there is a regular Borel measure $\nu_{x}$ on $\beta X$ such that $\operatorname{var}\left(\nu_{x}\right) \leq c \varepsilon$ and

$$
T f(x)=f(x)+\int_{\beta X} f d \nu_{x} \quad \text { for } f \in C(\beta X) .
$$

Let $K_{0} \in \mathcal{K}$ be such that $x \in K_{0}$. We show that the support of $\nu_{x}$ is contained in $K_{0}$. Assuming the contrary there is an $f_{0} \in C(\beta X)$ with $f_{0}=0$ on $K_{0}$ such that

$$
\int_{\beta X} f_{0} d \nu_{x}=1 \quad \text { and } \quad\left\|f_{0}\right\|_{X} \leq 1+\frac{1}{\left|\nu_{x}\right|\left(X \backslash K_{0}\right)} .
$$

Let $g_{0} \in C(\beta X)$ be such that $\left\|g_{0}\right\|_{X}=1$ and

$$
g_{0}=1 \quad \text { on } K_{0}, \quad g_{0}=0 \quad \text { on }\left\{t \in X:\left|f_{0}(t)\right|>\varepsilon\right\},
$$

so that

$$
\left\|f_{0} g_{0}\right\|_{X} \leq \varepsilon
$$

By Proposition 2, since $\left\|f_{0}\right\|_{K_{0}}=0$ we have $f_{0} \times g_{0}=0$ on $K_{0}$, and $\left\|f_{0} \times g_{0}\right\|_{X} \leq\left\|f_{0} g_{0}\right\|_{X}+\varepsilon\left\|f_{0}\right\|_{X}\left\|g_{0}\right\|_{X} \leq \varepsilon+\varepsilon\left\|f_{0}\right\|_{X} \leq \varepsilon\left(2+\frac{1}{\left|\nu_{x}\right|\left(X \backslash K_{0}\right)}\right)$. 
Hence

$$
\begin{aligned}
\left|T\left(f_{0} \times g_{0}\right)(x)\right| & =\left|\left(f_{0} \times g_{0}\right)(x)+\int_{\beta X}\left(f_{0} \times g_{0}\right) d \nu_{x}\right|=\left|\int_{\beta X \backslash K_{0}}\left(f_{0} \times g_{0}\right) d \nu_{x}\right| \\
& \leq\left|\nu_{x}\right|\left(X \backslash K_{0}\right)\left\|f_{0} \times g\right\|_{X} \leq\left|\nu_{x}\right|\left(X \backslash K_{0}\right) \varepsilon\left(2+\frac{1}{\left|\nu_{x}\right|\left(X \backslash K_{0}\right)}\right) \\
& \leq \varepsilon\left(2\left|\nu_{x}\right|\left(X \backslash K_{0}\right)+1\right) \leq \varepsilon(2 c \varepsilon+1) .
\end{aligned}
$$

On the other hand,

$$
\begin{aligned}
\left|T\left(f_{0} \times g_{0}\right)(x)\right| & =\left|T\left(f_{0}\right)(x) T\left(g_{0}\right)(x)\right| \\
& =\left|\int_{\beta X} f_{0} d \nu_{x}\right|\left|\left(1+\int_{\beta X} g_{0} d \nu_{x}\right)\right| \\
& \geq\left(1-\operatorname{var}\left(\nu_{x}\right)\right) \geq 1-c \varepsilon
\end{aligned}
$$

So

$$
\varepsilon(2 c \varepsilon+1) \geq 1-c \varepsilon,
$$

which is impossible provided $\varepsilon$ is small enough. We proved that

$$
\operatorname{supp}\left(\nu_{x}\right) \subset \bigcap\{K: x \in K \in \mathcal{K}\} \quad \text { for any } x \in X .
$$

Since a continuous function is bounded on any compact set, $\int_{\beta X} f d \nu_{x}$ is well defined for any $f \in C(X)$, and we can extend the map

$$
\mathrm{Id}+\Psi=T: C_{\mathrm{b}}(X) \rightarrow C_{\mathrm{b}}(X)
$$

to a map defined on all of $C(X)$. We shall use the same symbol $T$ to denote the extended map

$$
T(f)(x)=f(x)+\int_{\beta X} f d \nu_{x}, \quad f \in C(X) .
$$

To end the proof we shall show that $T$ is an algebra isomorphism from $(C(X), \times)$ onto $C(X)$; we need to check that $T$ is an algebra homomorphism, that it is injective, and that the range of $T$ consists of all continuous functions on $X$.

Let $f, g \in C(X)$. To show that

$$
T(f \times g)=T(f) T(g),
$$

fix $x \in X$ and $K \in \mathcal{K}$ with $x \in K$. Denote by $f_{1}, g_{1}, h_{1}$ arbitrary bounded continuous functions on $X$ that coincide on $K$ with $f, g$, and $f \times g$, respectively. By Proposition 2, since $\left\|f-f_{1}\right\|_{K}=\left\|g-g_{1}\right\|_{K}=0$, we have $\left\|f \times g-f_{1} \times g_{1}\right\|_{K}=0$, so since the support of $\nu_{x}$ is contained in $K$, by (4.5) we get 


$$
\begin{aligned}
T(f \times g)(x) & =(f \times g)(x)+\int_{\beta X}(f \times g) d \nu_{x}=\left(f_{1} \times g_{1}\right)(x)+\int_{\beta X}\left(f_{1} \times g_{1}\right) d \nu_{x} \\
& =T\left(f_{1} \times g_{1}\right)(x)=T\left(f_{1}\right)(x) T\left(g_{1}\right)(x) \\
& =\left(f_{1}(x)+\int_{\beta X} f_{1} d \nu_{x}\right)\left(g_{1}(x)+\int_{\beta X} g_{1} d \nu_{x}\right) \\
& =\left(f(x)+\int_{\beta X} f d \nu_{x}\right)\left(g(x)+\int_{\beta X} g d \nu_{x}\right)=T(f)(x) T(g)(x) .
\end{aligned}
$$

Now, to show that $T$ is injective, assume $f$ is a nonzero continuous function on $X$ such that $T(f)=0$. Select any compact set $K$ from $\mathcal{K}$ such that $f$ is not constantly zero on $K$, and let $y \in K$ be such that $\|f\|_{K}=|f(y)|>0$. Since $\operatorname{var}\left(\nu_{x}\right) \leq c \varepsilon$, for all $\varepsilon<1 / c$ we get

$$
0=T f(y)=\left|f(y)+\int_{K} f d \nu_{x}\right| \geq|f(y)|-c \varepsilon\|f\|_{K}=\|f\|_{K}(1-c \varepsilon)>0 .
$$

The contradiction shows that $T$ is injective.

It is clear that for any $f \in C(X)$ the function $T(f)$ is continuous on compact sets from $\mathcal{K}$. Since according to our assumptions $X$ is a $k$-space, it follows that $T(f)$ is continuous.

Define

$$
S(f)=\sum_{n=0}^{\infty}(-\Psi)^{n}(f) ;
$$

the map $S$ is an inverse of $T$ on the Banach algebra $C_{\mathrm{b}}(X)=C(\beta X)$. It is easy to check that for any $f \in C(X)$, the function $S(f)$ is well defined, that the value of $S(f)(x)$ depends only on the behavior of $f$ on $K$, for any $x \in K \in \mathcal{K}$, so $S(f) \in C(X)$, and consequently that $S=T^{-1}$, which proves that $T$ is surjective.

The last theorem shows that the algebras $C(X)$ are stable with respect to the most natural set of seminorms, maximum on compact subsets of $X$. As the next example shows, the same algebra with the same topology may not be stable with respect to some other seminorms.

Example 2. Put $A=C(\mathbb{R})$ with the usual pointwise multiplication, and

$$
p_{n}(f)=n \sup _{-n \leq t \leq n}|f(t)| \quad \text { for } f \in C(\mathbb{R}) \text { and } n \in \mathbb{N} .
$$

Fix $\varepsilon>0$ and define a new multiplication $\times$ on $A$ by

$$
(f \times g)(t)=(1+\varepsilon t) f(t) g(t), \quad t \in \mathbb{R} .
$$

It is easy to check that the new multiplication is an $\varepsilon$-deformation of the original one; however, the algebras $A$ and $(A, \times)$ are not isomorphic, as the first one has a unit while the second one does not. 
In the remainder of this section we will deal mostly with perturbations of topological algebras of analytic functions. The next two lemmas will provide us with technical tools; the first one gives a detailed analysis of a small deformations of the disc algebra. We denote by $A\left(\mathbb{D}_{R}\right)$ the Banach algebra of all functions that are analytic on $\mathbb{D}_{R}:=\{z \in \mathbb{C}:|z|<R\}$ and continuous on $\overline{\mathbb{D}}_{R}$; we equip it with the standard sup norm $\|f\|=\sup \{|f(z)|:|z| \leq R\}$ and pointwise multiplication.

Lemma 1. There is an $\varepsilon_{0}>0$ such that for any positive $\varepsilon<\varepsilon_{0}$, any $R>0$, and any multiplication $\times$ on $A\left(\mathbb{D}_{R}\right)$ with

$$
\|f \cdot g-f \times g\| \leq \varepsilon\|f\|\|g\|, \quad f \in A\left(\mathbb{D}_{R}\right),
$$

there is an algebra isomorphism $T_{R}$ from $\left(A\left(\mathbb{D}_{R}\right), \times\right)$ onto $A\left(\mathbb{D}_{R}\right)$ and a homeomorphism $\psi_{R}$ from $\overline{\mathbb{D}}_{R}$ onto itself such that

$$
\begin{gathered}
\left\|T_{R}\right\| \leq 1+\varepsilon, \quad\left\|T_{R}^{-1}\right\| \leq \frac{1}{1-\varepsilon}, \\
\left|T(f)\left(\psi_{R}(z)\right)-f(z)\right| \leq 2 \varepsilon, \quad z \in \overline{\mathbb{D}}_{R}, \\
(1-\varepsilon)|z| \leq\left|T_{R}(Z)(z)\right| \leq(1+\varepsilon)|z|, \quad z \in \overline{\mathbb{D}}_{R},
\end{gathered}
$$

where $Z$ is the identity function $Z(z)=z$. Moreover, if the functional $A\left(\mathbb{D}_{R}\right) \ni f \mapsto f(0)$ is $\times$-multiplicative, then also

$$
(1-\varepsilon)|z| \leq\left|T_{R}^{-1}(Z)(z)\right| \leq(1+\varepsilon)|z|, \quad z \in \overline{\mathbb{D}}_{R},
$$

and if $f_{0} \in A\left(\mathbb{D}_{R}\right)$ satisfies

$$
(1-\varepsilon)|z| \leq\left|f_{0}(z)\right| \leq(1+\varepsilon)|z|, \quad z \in \overline{\mathbb{D}}_{R},
$$

then

$$
(1-\varepsilon)^{2}|z| \leq\left|T_{R}\left(f_{0}\right)(z)\right| \leq(1+\varepsilon)^{2}|z|, \quad z \in \overline{\mathbb{D}}_{R} .
$$

Proof. Since the algebra $A\left(\mathbb{D}_{R}\right)$ is isometrically isomorphic to the stable disc algebra [20], there is an algebra isomorphism $T$ from $\left(A\left(\mathbb{D}_{R}\right), \times\right)$ onto $A\left(\mathbb{D}_{R}\right)$. We show that any such map has the remaining properties listed in the lemma, or may be modified to satisfy them. The property (4.8) follows directly from the proof of stability of the disc algebra (Lemma 3.3 of [20]). Basing on Remark 1 we can assume that both multiplications have the same unit 1.

For any $f \in A\left(\mathbb{D}_{R}\right)$ we have

$$
(1-\varepsilon)\|f\|^{2} \leq\|f \times f\| \leq(1+\varepsilon)\|f\|^{2},
$$

so for any $k \in \mathbb{N}$,

$$
(1-\varepsilon)^{2^{k}-1}\|f\|^{2^{k}} \leq\|\underbrace{f \times \ldots \times f}_{2^{k} \text { times }}\| \leq(1+\varepsilon)^{2^{k}-1}\|f\|^{2^{k}}
$$


and consequently the spectral radius $\varrho_{\times}(f)$ of $f$ in $\left(A\left(\mathbb{D}_{R}\right), \times\right)$ satisfies

$$
(1-\varepsilon)\|f\| \leq \varrho_{\times}(f) \leq(1+\varepsilon)\|f\| .
$$

Since $\varrho_{\times}(f)=\|T(f)\|$, we get

$$
\|T\| \leq 1+\varepsilon, \quad\left\|T^{-1}\right\| \leq \frac{1}{1-\varepsilon} .
$$

We now show that $Z \times A\left(\mathbb{D}_{R}\right)$ is a closed maximal ideal in $A\left(\mathbb{D}_{R}\right)$. Let $S: A\left(\mathbb{D}_{R}\right) \rightarrow A\left(\mathbb{D}_{R}\right)$ be defined by

$$
S(f)=T\left(\frac{f-f(0)}{Z}\right) T(Z)+f(0) .
$$

Notice that since the function $f-f(0)$ vanishes at zero, $(f-f(0)) / Z$ is a well defined element of $A\left(\mathbb{D}_{R}\right)$. Furthermore since $\|f-f(0)\| \leq 2\|f\|$, and $|Z|=R$ on the boundary of $\mathbb{D}_{R}$, we have

$$
\left|\frac{f-f(0)}{Z}(z)\right| \leq \frac{2\|f\|}{R} \quad \text { for } z \in \partial \mathbb{D}_{R},
$$

and consequently for all $z$ in $\mathbb{D}_{R}$, so

$$
\left\|\frac{f-f(0)}{Z}\right\| \leq \frac{2\|f\|}{R}
$$

As both multiplications have the same unit we have $T(\mathbf{1})=\mathbf{1}$ and

$$
\begin{aligned}
\left\|\left(T^{-1} \circ S\right)(f)-f\right\| & =\left\|T^{-1}\left(T\left(\frac{f-f(0)}{Z}\right) T(Z)\right)-\frac{f-f(0)}{Z} Z\right\| \\
& =\left\|\frac{\|-f(0)}{Z} \times Z-\frac{f-f(0)}{Z} Z\right\| \\
& \leq \varepsilon \frac{2\|f\|}{R} R=2 \varepsilon\|f\| .
\end{aligned}
$$

Hence, provided $2 \varepsilon<1$, the map $T^{-1} \circ S$, and consequently $S$, is invertible. Since $Z A\left(\mathbb{D}_{R}\right)$ is a codimension one closed subspace of $A\left(\mathbb{D}_{R}\right)$, so is its image under $S$. Hence $T\left(Z \times A\left(\mathbb{D}_{R}\right)\right)=T(Z) A\left(\mathbb{D}_{R}\right)=S\left(Z A\left(\mathbb{D}_{R}\right)\right)$ is a maximal ideal in $A\left(\mathbb{D}_{R}\right)$. Consequently, there is a $z_{0} \in \overline{\mathbb{D}}_{R}$ such that $T(Z) A\left(\mathbb{D}_{R}\right)=$ $\left\{f \in A\left(\mathbb{D}_{R}\right): f\left(z_{0}\right)=0\right\}$, and the point $z_{0}$ cannot be in the boundary of $\mathbb{D}_{R}$, since the corresponding ideal is principal. Put

$$
B_{z_{0}}(z)=\frac{R z_{0}-z}{R-z \bar{z}_{0}}
$$

and define $T_{R}: A\left(\mathbb{D}_{R}\right) \rightarrow A\left(\mathbb{D}_{R}\right)$ by

$$
T_{R}(f)=T(f) \circ B_{z_{0}}, \quad f \in A\left(\mathbb{D}_{R}\right) .
$$

The new map $T_{R}$ still has all the properties of $T$, that is, it satisfies (4.7) and is an algebra isomorphism from $\left(A\left(\mathbb{D}_{R}\right), \times\right)$ onto $A\left(\mathbb{D}_{R}\right)$; in addition,

$$
T_{R}\left(Z \times A\left(\mathbb{D}_{R}\right)\right)=T_{R}(Z) A\left(\mathbb{D}_{R}\right)=\left\{f \in A\left(\mathbb{D}_{R}\right): f(0)=0\right\} .
$$


Hence $T_{R}(Z)$ has only one single zero, so $g_{0}:=T_{R}(Z) / Z$ is an invertible element of $A\left(\mathbb{D}_{R}\right)$. Since

$$
\left\|g_{0}\right\|=\left\|\frac{T_{R}(Z)}{Z}\right\|=\frac{\left\|T_{R}(Z)\right\|}{R} \leq \frac{\left\|T_{R}\right\|\|Z\|}{R}=\left\|T_{R}\right\| \leq 1+\varepsilon
$$

we get

$$
\left|T_{R}(Z)(z)\right| \leq(1+\varepsilon)|z|, \quad z \in \mathbb{D}_{R}
$$

Since $T_{R}(Z) / Z$ is invertible, its absolute value attains its minimum on the boundary of $\mathbb{D}_{R}$. Assume there is a point $w_{0} \in \partial \mathbb{D}_{R}$ such that $\left\|T_{R}(Z) / R\right\|=$ $\left\|T_{R}(Z) / Z\right\|=\left|\left(T_{R}(Z) / Z\right)\left(w_{0}\right)\right|<1-\varepsilon$ and let $h_{0} \in A\left(\mathbb{D}_{R}\right)$ be a norm one function such that $\left\|h_{0} T_{R}(Z) / R\right\|<1-\varepsilon$. We have

$$
\begin{aligned}
1-\varepsilon & >\left\|h_{0} \frac{T_{R}(Z)}{R}\right\| \geq(1-\varepsilon)\left\|T_{R}^{-1}\left(h_{0} \frac{T_{R}(Z)}{R}\right)\right\| \\
& =\left\|h_{0} \times \frac{Z}{R}\right\| \geq\left\|h_{0} \frac{Z}{R}\right\|-\left\|h_{0} \times \frac{Z}{R}-h_{0} \frac{Z}{R}\right\| \geq 1-\varepsilon .
\end{aligned}
$$

The contradiction shows that

$$
\left\|g_{0}^{-1}\right\|=\left\|\frac{Z}{T_{R}(Z)}\right\| \leq \frac{1}{1-\varepsilon},
$$

so $(1-\varepsilon)|z| \leq\left|T_{R}(Z)(z)\right|$ for $z \in \overline{\mathbb{D}}_{R}$.

Assume now that the functional $A\left(\mathbb{D}_{R}\right) \ni f \mapsto f(0)$ is $\times$-multiplicative and put $h_{0}=T_{R}^{-1}\left(g_{0}^{-1}\right)$. We have

$$
T_{R}^{-1}(Z)(0)=T_{R}^{-1}\left(g_{0}^{-1} T_{R}(Z)\right)(0)=h_{0} \times Z(0)=0
$$

and

$$
\left\|\frac{T_{R}^{-1}(Z)}{Z}\right\| \leq \frac{\left\|T_{R}^{-1}\right\|\|Z\|}{R} \leq 1+\varepsilon,
$$

hence

$$
\left|T_{R}^{-1}(Z)(z)\right| \leq(1+\varepsilon)|z|, \quad z \in \overline{\mathbb{D}}_{R} .
$$

Using arguments very similar to those before one can now show that $T_{R}^{-1}(Z) A\left(\mathbb{D}_{R}\right)$ is a closed maximal ideal in $A\left(\mathbb{D}_{R}\right)$, so $T_{R}^{-1}(Z) / Z$ is invertible with

$$
\left\|\frac{Z}{T_{R}^{-1}(Z)}\right\| \leq \frac{1}{1-\varepsilon},
$$

and consequently

$$
(1-\varepsilon)|z| \leq\left|T_{R}^{-1}(Z)(z)\right|, \quad z \in \overline{\mathbb{D}}_{R} .
$$

Assume $f_{0} \in A\left(\mathbb{D}_{R}\right)$ satisfies

$$
(1-\varepsilon)|z| \leq\left|f_{0}(z)\right| \leq(1+\varepsilon)|z|, \quad z \in \mathbb{D}_{R} .
$$


If we replace $Z$ with $f_{0}$ in the first part of our proof we get

$$
\left\|\frac{T_{R}\left(f_{0}\right)}{Z}\right\| \leq(1+\varepsilon)^{2} \quad \text { and } \quad\left\|\frac{Z}{T_{R}\left(f_{0}\right)}\right\| \leq \frac{1}{(1-\varepsilon)^{2}},
$$

so (4.9) follows.

Lemma 2. Let $\mathcal{F}$ be a free ultrafilter on the set $\mathbb{N}$ of natural numbers, let $r<R$ be positive real numbers, and let $f_{n}$ be a sequence of holomorphic functions on $\mathbb{D}_{R}$ such that $\sup _{n \in \mathbb{N}}\left\|f_{n}\right\|_{R}<\infty$. Then $\lim _{\mathcal{F}} f_{n}(z)$ is holomorphic on $\mathbb{D}_{R}$ and $\lim _{\mathcal{F}}\left\|f_{n}\right\|_{r}=\left\|\lim _{\mathcal{F}} f_{n}\right\|_{r}$.

Notice that if we only assumed that $f_{n}$ were continuous then the limit $\lim _{\mathcal{F}} f_{n}(z)$ could be discontinuous and $\left\|\lim _{\mathcal{F}} f_{n}\right\|_{r}$ could be strictly smaller than $\lim _{\mathcal{F}}\left\|f_{n}\right\|_{r}$. We refer to [4] for a review of basic applications of ultrafilters in the Banach space theory (see also [2]).

Proof of Lemma 2. Let $z_{0}$ be a point in $\mathbb{D}_{R}$. We show that $f(z):=$ $\lim _{\mathcal{F}} f_{n}(z)$ is holomorphic at $z_{0}$ and $\lim _{\mathcal{F}}\left\|f_{n}\right\|_{r}=\left\|\lim _{\mathcal{F}} f_{n}\right\|_{r}$. To simplify the notation we can assume without loss of generality that

- $z_{0}=0$ (compose all $f_{n}$ with a suitable holomorphic transformation of $\mathbb{D}_{R}$ onto itself mapping 0 onto $\left.z_{0}\right)$,

- $f_{n}(0)=0$ for $n \in \mathbb{N}$,

- $f_{n}^{\prime}(0)=0$ for $n \in \mathbb{N}$ (subtract the bounded sequence $\left.f_{n}^{\prime}(0) z\right)$,

- $R>1>r$ and $\sup _{n \in \mathbb{N}}\left\|f_{n}\right\|_{R} \leq 1$.

For any $z \in \mathbb{D}_{r}$ we have

$$
\left|f_{n}^{\prime}(z)\right| \leq\left|\frac{1}{2 \pi} \oint_{|\xi|=1} \frac{f_{n}(\xi)}{(\xi-z)^{2}} d \xi\right| \leq \frac{4}{(1-r)^{2}},
$$

so $\left|f_{n}(z)\right| \leq 4(1-r)^{-2}|z|$, hence $\left|\lim _{\mathcal{F}} f_{n}(z)\right| \leq 4(1-r)^{-2}|z|$ and $\left(\lim _{\mathcal{F}} f_{n}\right)^{\prime}(0)$ $=0$.

Assume $s$ is such that $\left\|\lim _{\mathcal{F}} f_{n}\right\|_{r}<s<\lim _{\mathcal{F}}\left\|f_{n}\right\|_{r}$ and let $U=$ $\left\{U_{1}, \ldots, U_{p}\right\}$ be a finite cover of $\mathbb{D}_{r}$ consisting of nonempty sets with diameter less than $\frac{1}{8}(1-r)^{2}\left(s-\left\|\lim _{\mathcal{F}} f_{n}\right\|_{r}\right)$. For any $j$ fix $w_{j} \in U_{j}$. For arbitrary $n \in \mathbb{N}$ and $w \in U_{j}$ we have

$$
\begin{aligned}
\left|f_{n}\left(w_{j}\right)-f_{n}(w)\right| & \leq \sup _{z \in U_{j}}\left|f_{n}^{\prime}(z)\right|\left|w_{j}-w\right| \\
& \leq \frac{4}{(1-r)^{2}} \cdot \frac{(1-r)^{2}}{8}\left(s-\left\|\lim _{\mathcal{F}} f_{n}\right\|_{r}\right) \\
& =\frac{s-\left\|\lim _{\mathcal{F}} f_{n}\right\|_{r}}{2} .
\end{aligned}
$$

Put $\mathbb{N}_{j}=\left\{k \in \mathbb{N}: \sup _{z \in U_{j}}\left|f_{k}(z)\right|>s\right\}$. Since $\bigcup_{j=1}^{p} \mathbb{N}_{j}$ contains all but finitely many natural numbers and $\mathcal{F}$ is a free ultrafilter there is a $j_{0}$ such 
that $\mathbb{N}_{j_{0}} \in \mathcal{F}$. We have

$$
\begin{aligned}
\left\|\lim _{\mathcal{F}} f_{n}\right\|_{r} & \geq\left|\lim _{\mathcal{F}} f_{n}\left(w_{j_{0}}\right)\right|=\lim _{\mathcal{F}}\left|f_{n}\left(w_{j_{0}}\right)\right| \\
& \geq \lim _{\mathcal{F}}\left(\sup _{w \in U_{j_{0}}}|f(w)|-\sup _{w \in U_{j_{0}}}\left|f_{n}\left(w_{j_{0}}\right)-f_{n}(w)\right|\right) \\
& \geq s-\frac{s-\left\|\lim _{\mathcal{F}} f_{n}\right\|_{r}}{2}>\left\|\lim _{\mathcal{F}} f_{n}\right\|_{r} .
\end{aligned}
$$

The contradiction shows that $\lim _{\mathcal{F}}\left\|f_{n}\right\|_{r}=\left\|\lim _{\mathcal{F}} f_{n}\right\|_{r}$.

THEOREM 4. Let $A=\operatorname{Hol}(\mathbb{D})$ be the algebra of all holomorphic functions on the open unit disc $\mathbb{D}$ with the usual pointwise multiplication, let $r_{n}$ be an increasing sequence of positive numbers with $\lim r_{n}=1$, and let

$$
\|f\|_{n}=\sup \left\{|f(z)|:|z| \leq r_{n}\right\} \text {. }
$$

Then the topological algebra $\left(A,\left\{\|\cdot\|_{n}: n=1,2,3, \ldots\right\}\right)$ is stable.

Proof. Fix an $\varepsilon>0$ and assume $\times$ is another multiplication on $A$ such that for all $n \in \mathbb{N}$,

$$
\|f \cdot g-f \times g\|_{n} \leq \varepsilon\|f\|_{n}\|g\|_{n}, \quad f, g \in A .
$$

Put $A_{n}:=A\left(\mathbb{D}_{r_{n}}\right), n \in \mathbb{N}, A_{\infty}:=A(\mathbb{D})$, and let $\|\cdot\|:=\sup \|\cdot\|_{n}$ be the usual sup norm on $\mathbb{D}$. Since $A$ is dense in $A_{n}$ and the multiplication $\times$ is jointly continuous, it can be uniquely extended to a multiplication on $A_{n}$, $n \in \mathbb{N}$; the extension still satisfies (4.10). We also have

$$
\|f \cdot g-f \times g\| \leq \varepsilon\|f\|\|g\|, \quad f, g \in A_{\infty} .
$$

Let $T_{n}, n \in \mathbb{N} \cup\{\infty\}$, be an isomorphism from $\left(A_{n}, \times\right)$ onto $\left(A_{n}, \cdot\right)$, given by Lemma 1 . We shall show that $T_{\infty}$ can be extended to an isomorphism from $(A, \times)$ onto $(A, \cdot)$.

Let $n_{1}<n_{2} \in \mathbb{N} \cup\{\infty\}$. The composition map $T_{n_{1}} \circ T_{n_{2}}^{-1}: A_{n_{2}} \rightarrow A_{n_{1}}$ is a homeomorphism between two uniform Banach algebras so it must be given by a continuous map $\varphi_{n_{1}, n_{2}}: \overline{\mathbb{D}}_{r_{n_{1}}} \rightarrow \overline{\mathbb{D}}_{r_{n_{2}}}$ between the maximal ideal spaces of these algebras:

Hence

$$
\left(T_{n_{1}} \circ T_{n_{2}}^{-1}\right)(f)=f \circ \varphi_{n_{1}, n_{2}}, \quad f \in A_{n_{2}} .
$$

$$
T_{\infty}(f)\left(\varphi_{n_{1}, \infty}(z)\right)=T_{n_{1}}(f)(z), \quad f \in A_{\infty}, z \in \overline{\mathbb{D}}_{r_{n_{1}}} .
$$

Since $A_{n_{2}}$ is dense in $A_{n_{1}},\left(T_{n_{1}} \circ T_{n_{2}}^{-1}\right)\left(A_{n_{2}}\right)$ is dense in $A_{n_{1}}$ and must separate points of $\overline{\mathbb{D}}_{r_{n_{1}}}$, the maximal ideal space of $A_{n_{1}}$, so $\varphi_{n_{1}, n_{2}}$ is injective; moreover $\varphi_{n_{1}, n_{2}}=\left(T_{n_{2}} \circ T_{n_{1}}^{-1}\right)(Z)$. Notice also that

$$
\varphi_{n_{1}, \infty}(z)=\left(\varphi_{n_{2}, \infty} \circ \varphi_{n_{2}, n_{1}}\right)(z) \quad \text { for } z \in \overline{\mathbb{D}}_{r_{n_{1}}} \text {. }
$$

We show that $\mathbb{D}=\bigcup_{n=1}^{\infty} \varphi_{n, \infty}\left(\overline{\mathbb{D}}_{r_{n}}\right)$. Assume again that $n_{1}<n_{2}$. Since $\overline{\mathbb{D}}_{r_{n_{1}}}$ is a compact subset of $\overline{\mathbb{D}}_{r_{n_{2}}}$ and $\varphi_{n_{2}, n_{1}}$ is injective it follows that $\varphi_{n_{2}, n_{1}}\left(\overline{\mathbb{D}}_{r_{n_{1}}}\right)$ 
is compact and contained in $\overline{\mathbb{D}}_{r_{n_{2}}}$ so $\varphi_{n_{1}, \infty}\left(\overline{\mathbb{D}}_{r_{n_{1}}}\right)=\varphi_{n_{2}, \infty}\left(\varphi_{n_{2}, n_{1}}\left(\overline{\mathbb{D}}_{r_{n_{1}}}\right)\right) \subset \mathbb{D}$. Hence $\bigcup_{n=1}^{\infty} \varphi_{n, \infty}\left(\overline{\mathbb{D}}_{r_{n}}\right) \subset \mathbb{D}$.

To show the other inclusion let $z_{0} \in \mathbb{D}$. Put

$$
B_{z_{0}}(w)=\frac{z_{0}-w}{1-\bar{z}_{0} w}, \quad z \in \mathbb{D} .
$$

Let $\psi_{1}, \psi_{r_{k}}$ be as in Lemma 1 . We have

$$
\left\|\left(T_{k} \circ T_{\infty}^{-1}\right)\left(B_{z_{0}}\right)-T_{\infty}^{-1}\left(B_{z_{0}}\right) \circ \psi_{r_{k}}^{-1}\right\| \leq 2 \varepsilon
$$

and

$$
\left\|T_{\infty}^{-1}\left(B_{z_{0}}\right) \circ \psi_{r_{k}}^{-1}-B_{z_{0}} \circ \psi_{1} \circ \psi_{r_{k}}^{-1}\right\| \leq 2 \varepsilon \frac{1+\varepsilon}{1-\varepsilon} .
$$

Hence, assuming $r_{k}$ is close to 1 , the function $B_{z_{0}} \circ \psi_{1} \circ \psi_{r_{k}}^{-1}$ is a homeomorphism from $\overline{\mathbb{D}}_{r_{k}}$ onto a subset of the plane very close to $\overline{\mathbb{D}}_{r_{k}}$. Such a set must contain 0 , so there is a $w_{0} \in \overline{\mathbb{D}}_{r_{k}}$ with $\left(T_{k} \circ T_{\infty}^{-1}\right)\left(B_{z_{0}}\right)\left(w_{0}\right)=0$.

We have

$$
\begin{aligned}
\left\{f \in A_{\infty}: T_{\infty}(f)\left(z_{0}\right)=0\right\} & =T_{\infty}^{-1}\left(B_{z_{0}} A_{\infty}\right)=T_{\infty}^{-1}\left(B_{z_{0}}\right) \times A_{\infty} \\
& \subset A_{\infty} \cap T_{k}^{-1}\left(T_{k} T_{\infty}^{-1}\left(B_{z_{0}}\right) \times A_{k}\right. \\
& =A_{\infty} \cap T_{k}^{-1}\left(T_{k} T_{\infty}^{-1}\left(B_{z_{0}}\right) A_{k}\right) \\
& \subset\left\{f \in A_{\infty}: T_{k}(f)\left(w_{0}\right)=0\right\} .
\end{aligned}
$$

Since the codimension of the first and the last ideal above is the same, they must be identical and consequently the corresponding $\times$-multiplicative functionals on $A_{\infty}$ must coincide, that is,

$$
T_{\infty}(f)\left(z_{0}\right)=T_{k}(f)\left(w_{0}\right) \quad \text { for } f \in A_{\infty} .
$$

By (4.11) we get $\varphi_{k, \infty}\left(w_{0}\right)=z_{0}$, which shows that $\mathbb{D} \subset \bigcup_{n=1}^{\infty} \varphi_{n, \infty}\left(\overline{\mathbb{D}}_{r_{n}}\right)$.

To end the proof fix $n_{0}$ and let $j_{0}, j_{1}$ be such that $\overline{\mathbb{D}}_{r_{n_{0}}} \subset \varphi_{j_{0}, \infty}\left(\overline{\mathbb{D}}_{r_{j_{0}}}\right)$ and $\varphi_{j_{0}, \infty}\left(\overline{\mathbb{D}}_{r_{n_{0}}}\right) \subset \overline{\mathbb{D}}_{r_{j_{1}}}$. For any $f \in A_{0}$ we have

$$
\begin{aligned}
\left\|T_{\infty}(f)\right\|_{n_{0}} & =\sup \left\{\left|T_{0}(f)(z)\right|: z \in \overline{\mathbb{D}}_{r_{n_{0}}}\right\} \leq \sup \left\{\left|T_{\infty}(f)(z)\right|: z \in \varphi_{j_{0}, \infty}\left(\overline{\mathbb{D}}_{r_{j_{0}}}\right)\right\} \\
& =\sup \left\{\left|T_{j_{0}}(f)(z)\right|: z \in \mathbb{D}_{r_{j_{0}}}\right\}=\left\|T_{j_{0}}(f)\right\|_{j_{0}} \leq(1+\varepsilon)\|f\|_{j_{0}},
\end{aligned}
$$

and

$$
\begin{aligned}
\|f\|_{n_{0}} & =\sup \left\{|f(z)|: z \in \overline{\mathbb{D}}_{r_{n_{0}}}\right\} \leq \sup \left\{|f(z)|: z \in \varphi_{j_{0}, \infty}\left(\overline{\mathbb{D}}_{r_{j_{1}}}\right)\right\} \\
& =\sup \left\{\left|T_{j_{1}} \circ T_{\infty}^{-1}(f)(z)\right|: z \in \mathbb{D}_{r_{j_{1}}}\right\}=\left\|T_{j_{0}}\left(T_{\infty}^{-1}(f)\right)\right\|_{j_{1}} \leq \frac{1}{1-\varepsilon}\left\|T_{\infty}^{-1}(f)\right\|_{j_{1}} .
\end{aligned}
$$

The above shows that $T_{\infty}$ and $T_{\infty}^{-1}$ are continuous in the topology of $\left(A,\left\{\|\cdot\|_{n}: n=1,2,3, \ldots\right\}\right)$ so $T_{\infty}$ can be extended to a homomorphism from the algebra $\left(A, \times,\left\{\|\cdot\|_{n}: n=1,2,3, \ldots\right\}\right)$ onto $\left(A, \cdot,\left\{\|\cdot\|_{n}: n=1,2,3, \ldots\right\}\right)$ as promised. 
TheOREM 5. Let $A=\operatorname{Hol}(\mathbb{C})$ be the algebra of all holomorphic functions on $\mathbb{C}$ with the usual pointwise multiplication, let $k_{n}$ be an increasing sequence of positive numbers with $\lim k_{n}=\infty$, and let

$$
\|f\|_{n}=\sup \left\{|f(z)|:|z| \leq k_{n}\right\} .
$$

Then the topological algebra $\left(A,\left\{\|\cdot\|_{n}: n=1,2,3, \ldots\right\}\right)$ is stable.

Notice that in contrast to Theorem 4 , this time the subalgebra $\{f \in A$ : $\left.\sup _{n}\|f\|_{n}<\infty\right\}$ is trivial and cannot be helpful in the proof.

Proof. Without loss of generality, discarding some of the norms $\|\cdot\|_{n}$ if necessary, we may assume that

$$
k_{n} / k_{n+1} \leq(1-\varepsilon)^{2} \quad \text { for } n \in \mathbb{N} .
$$

Fix an $\varepsilon>0$ and assume $\times$ is another multiplication on $A$ such that for all $n \in \mathbb{N}$,

$$
\|f \cdot g-f \times g\|_{n} \leq \varepsilon\|f\|_{n}\|g\|_{n} \quad f, g \in A .
$$

Since $A$ is dense in $A_{n}:=A\left(\mathbb{D}_{k_{n}}\right), n \in \mathbb{N}$, the new multiplication $\times$ can be uniquely extended to a multiplication on $A_{n}$; the extension still satisfies (4.12). Let $T_{n}$ be the isomorphism from $\left(A_{n}, \times\right)$ onto $\left(A_{n}, \cdot\right)$ given by Lemma 1 . By the same lemma there is a $\times$-multiplicative functional $F$ on $A_{1}$ defined by $F(f):=T_{1}(f)\left(\psi_{k_{1}}(0)\right)$ such that $\|F(f)-f(0)\| \leq 2 \varepsilon\|f\|$. Let $\mu$ be a measure on $\overline{\mathbb{D}}_{k_{1}}$ such that

$$
\operatorname{var}(\mu) \leq 2 \varepsilon \quad \text { and } \quad \int_{\overline{\mathbb{D}}_{k_{1}}} f d \mu=F(f)-f(0) \quad \text { for } f \in A_{1} .
$$

Put

$$
\Phi: A \rightarrow A, \quad \Phi(f)=f+\int_{\overline{\mathbb{D}}_{k_{1}}} f d \mu,
$$

and define another multiplication $\times^{\prime}$ on $A$ by

$$
f \times^{\prime} g=\Phi\left(\Phi^{-1}(f) \times \Phi^{-1}(g)\right) .
$$

Since $\Phi(f)(0)=F(f)$ and $F$ is $\times$-multiplicative we get

$$
\begin{aligned}
\left(f \times^{\prime} g\right)(0) & =F\left(\Phi^{-1}(f) \times \Phi^{-1}(g)\right)=\left(F \circ \Phi^{-1}\right)(f) F \circ \Phi^{-1}(g) \\
& =\Phi\left(\Phi^{-1}(f)\right)(0) \Phi\left(\Phi^{-1}(g)\right)(0)=f(0) g(0) .
\end{aligned}
$$

Hence the algebras $(A, \times)$ and $\left(A, \times^{\prime}\right)$ are topologically isomorphic $(\Phi$ is an isomorphism), the new multiplication $x^{\prime}$ is a small deformation of $\times$, so it is also a small deformation of the original multiplication - on $A$, and the evaluation at 0 is $\times^{\prime}$-multiplicative. Consequently, it is enough to show that the algebras $A$ and $\left(A, \times^{\prime}\right)$ are isomorphic; in order to simplify the notation we will just assume that the evaluation at 0 is already $\times$-multiplicative. 
Let $\mathcal{F}$ be a free ultrafilter on $\mathbb{N}$ and define $T: A \rightarrow A$ by

$$
T(f)(z)=\left(\lim _{\mathcal{F}} T_{n}(f)\right)(z), \quad f \in A .
$$

We need to show that $\lim _{\mathcal{F}} T_{n}(f)$ is a well defined element of $A$, and that $T$ is a bijective algebra isomorphism from $(A, \times)$ onto $A$.

Assume $n_{2}>n_{1}>n_{0}$. The composition map $T_{n_{1}} \circ T_{n_{2}}^{-1}: A_{n_{2}} \rightarrow A_{n_{1}}$ is a homeomorphism between two uniform Banach algebras, so must be given by a continuous map $\varphi_{n_{1}, n_{2}}: \overline{\mathbb{D}}_{k_{n_{1}}} \rightarrow \overline{\mathbb{D}}_{k_{n_{2}}}$ between the maximal ideal spaces of these algebras:

$$
\left(T_{n_{1}} \circ T_{n_{2}}^{-1}\right)(f)=f \circ \varphi_{n_{1}, n_{2}}, \quad f \in A_{n_{2}} .
$$

Hence

$$
T_{n_{2}}(f)\left(\varphi_{n_{1}, n_{2}}(z)\right)=T_{n_{1}}(f)(z), \quad f \in A_{n_{2}}, \quad z \in \overline{\mathbb{D}}_{k_{n_{1}}} .
$$

Since $A_{n_{2}}$ is dense in $A_{n_{1}}$, and $T_{n_{i}}$ are isomorphisms, $\left(T_{n_{1}} \circ T_{n_{2}}^{-1}\right)\left(A_{n_{2}}\right)$ is dense in $A_{n_{1}}$ and must separate points of $\overline{\mathbb{D}}_{k_{n_{1}}}$, the maximal ideal space of $A_{n_{1}}$, so $\varphi_{n_{1}, n_{2}}$ is injective.

Since $\varphi_{n_{1}, n_{2}}=\left(T_{n_{1}} \circ T_{n_{2}}^{-1}\right)(Z)$, by Lemma 1 with $f_{0}=T_{n_{2}}^{-1}(Z)$ we get

$$
(1-\varepsilon)^{2}|z| \leq\left|\varphi_{n_{1}, n_{2}}(z)\right| \leq(1+\varepsilon)^{2}|z|, \quad z \in \overline{\mathbb{D}}_{k_{n_{1}}} .
$$

Let $f \in A$ and $|z| \leq k_{n_{0}} \leq(1-\varepsilon)^{2} k_{n_{1}}$ so that $z \in \varphi_{n_{1}, n_{2}}\left(\overline{\mathbb{D}}_{k_{n_{1}}}\right)$. Then

$$
\begin{aligned}
& \left\|T_{n_{2}}(f)\right\|_{n_{0}} \leq \sup _{w \in \overline{\mathbb{D}}_{k_{n_{1}}}\left|T_{n_{2}}(f)\left(\varphi_{n_{1}, n_{2}}(w)\right)\right|}=\sup _{w \in \overline{\mathbb{D}}_{k_{n_{1}}}\left|T_{n_{1}}(f)(z)\right| \leq(1+\varepsilon)\|f\|_{n_{1}},}, \\
& \left\|T_{n_{2}}(f)\right\|_{n_{2}} \geq \sup _{w \in \overline{\mathbb{D}}_{k_{n_{1}}}}\left|T_{n_{2}}(f)\left(\varphi_{n_{1}, n_{2}}(w)\right)\right|=\sup _{w \in \overline{\mathbb{D}}_{k_{n_{1}}}}\left|T_{n_{1}}(f)(z)\right| \geq(1-\varepsilon)\|f\|_{n_{1}} .
\end{aligned}
$$

Hence by Lemma $2, \lim _{\mathcal{F}} T_{n}(f)$ is a well defined analytic function and

$$
(1-\varepsilon)\|f\|_{k} \leq\left\|\lim _{\mathcal{F}} T_{n}(f)\right\|_{k+1} \leq(1+\varepsilon)\|f\|_{k+2} .
$$

So $T$ is a topological isomorphism from $(A, \times)$ onto a closed subalgebra of $A$. To show that $T$ is surjective it is enough to notice that $Z \in T(A)$. Indeed, $T(Z) / Z$ is a bounded entire function by Lemma 1 , so it is constant.

Theorem 6. Let $0<r<R<\infty$, let $A=\operatorname{Hol}(P)$ be the algebra of all holomorphic functions on $P=\{z \in \mathbb{C}: r<|z|<R\}$ with the usual pointwise multiplication, let $\left(k_{n}\right)_{n=-\infty}^{\infty}$ be an increasing sequence of positive numbers with $\lim _{n \rightarrow-\infty} k_{n}=r$ and $\lim _{n \rightarrow \infty} k_{n}=R$, and let

$$
\|f\|_{n}=\sup \left\{|f(z)|: k_{-n} \leq|z| \leq k_{n}\right\} .
$$

Then the topological algebra $\left(A,\left\{\|\cdot\|_{n}: n=1,2,3, \ldots\right\}\right)$ is not stable. 
Proof (sketch). Fix an $\varepsilon>0$, put $P_{\varepsilon}=\{z \in \mathbb{C}: r<|z|<(1+\varepsilon) R\}$ and define $T: A \rightarrow \operatorname{Hol}\left(P_{\varepsilon}\right)$ by

$$
\begin{aligned}
T\left(\sum_{n=-\infty}^{\infty} a_{n} Z^{n}\right)= & \sum_{n=-\infty}^{0} a_{n} Z^{n} \\
& +\sum_{n=-\infty}^{\infty} a_{n}\left(\frac{R}{R+\varepsilon}\right) Z^{n} \quad \text { for } \sum_{n=-\infty}^{\infty} a_{n} Z^{n} \in \operatorname{Hol}(P) .
\end{aligned}
$$

It is obvious that $T$ is a well defined linear bijection and that the algebras $\operatorname{Hol}(P)$ and $\operatorname{Hol}\left(P_{\varepsilon}\right)$ are not isomorphic since their maximal ideal spaces are not holomorphically homeomorphic. Hence

$$
f \times g:=T^{-1}(T(f) T(g))
$$

defines a new multiplication on $A$ such that $A$ and $(A, \times)$ are not isomorphic.

Put $P_{n}:=\left\{z: k_{-n} \leq|z| \leq k_{n}\right\}$ and $P_{n}^{\prime}:=\left\{z: k_{-n} \leq|z| \leq(1+\varepsilon) k_{n}\right\}$. Notice that for each $n$, the bijection $T$ maps $\left\{f \in C\left(P_{n}\right): f \in \operatorname{Hol}\left(\operatorname{int} P_{n}\right)\right\}$ onto $\left\{f \in C\left(P_{n}\right): f \in \operatorname{Hol}\left(\operatorname{int} P_{n}^{\prime}\right)\right\}$. One can verify that the norms of these maps, as well as the norms of their inverses tend to one uniformly as $\varepsilon \rightarrow 0$, and hence by Theorem 3.1 of [5],

$$
\|f \times g-f g\|_{n} \leq\left(1+\varepsilon^{\prime}\right)\|f\|_{n}\|g\|_{n} \quad \text { for all } f, g \in A \text { and } n \in \mathbb{N}
$$

where $\varepsilon^{\prime} \rightarrow 0$ as $\varepsilon \rightarrow 0$.

THEOREM 7. The property of being a Q-algebra is not stable.

Proof. Let $A$ be the space $C(\mathbb{C})$ of all continuous functions on the complex plane with the topology defined by the family $\Xi=\left\{\|\cdot\|_{n}: n=\right.$ $1,2,3, \ldots\}$, where

$$
\|f\|_{n}=\sup \{|f(z)|:|z| \leq n\}
$$

and let - be a zero multiplication on $A$, that is, $a \cdot b=0$ for all $a, b \in A$. It is immediate to check that $A$ is a $Q$-algebra.

Fix an $\varepsilon>0$ and define a new multiplication $\times$ on $A$ by

$$
(a \times b)(z)=\varepsilon a(z) b(z) \quad \text { for } a, b \in A, z \in \mathbb{C} .
$$

We have

$$
\|a \cdot b-a \times b\|_{n}=\|a \times b\|_{n} \leq \varepsilon\|a\|_{n}\|b\|_{n},
$$

so $\times$ is an $\varepsilon$-perturbation of the original multiplication of $A$. However $(A, \times)$ is not a $Q$-algebra since it has a unit (the constant function $1 / \varepsilon$ ), and any neighborhood

$$
\left\{f \in A:\|f-1 / \varepsilon\|_{n}<\delta\right\}
$$

of that unit contains functions equal zero at some point of the plane; such functions are not invertible in the algebra $(A, \times)$. 
Notice that all the elements of the algebra $(A, \cdot)$ considered in the last proof are bounded, but the identity function is not bounded in the algebra $(A, \times)$; hence we get the following proposition.

Proposition 3. The property of $m$-convex algebras of having bounded elements is not stable.

On the other hand the related uniform property is stable.

Proposition 4. The property of m-convex algebras of having uniformly bounded elements is stable.

Proof. Let $(A, \cdot, \Xi)$ be an $m$-convex algebra with uniformly bounded elements, where $\Xi=\left\{p_{\lambda}: \lambda \in \Lambda\right\}$ is a family of $m$-convex seminorms defining the topology of the algebra. Let $\times$ be an $\varepsilon$-deformation of $A$, and let $a$ be a fixed element of $A$.

Let $\mu_{a}$ and $M$ be positive numbers such that

$$
p_{\lambda}\left(\left(a / \mu_{a}\right)^{n}\right) \leq M \quad \text { for all } n \in \mathbb{N} \text { and } \lambda \in \Lambda .
$$

We get

$$
\begin{aligned}
p_{\lambda}(\underbrace{\frac{a}{M(1+\varepsilon) \mu_{a}} \times \ldots \times \frac{a}{M(1+\varepsilon) \mu_{a}}}_{n \text { times }}) & \leq(1+\varepsilon)^{n-1}\left[p_{\lambda}\left(\frac{a}{M(1+\varepsilon) \mu_{a}}\right)\right]^{n} \\
& =\frac{1}{M^{n}(1+\varepsilon)}\left[p_{\lambda}\left(\frac{a}{\mu_{a}}\right)\right]^{n} \leq \frac{1}{1+\varepsilon}<1 .
\end{aligned}
$$

Hence the elements of $(A, \times)$ are uniformly bounded.

5. Almost multiplicative functionals and other problems. Small deformations of multiplication are not the only aspect of the deformation theory of Banach algebras that one may try to extend to topological algebras. We would like to mention briefly some other natural problems.

The first one concerns almost multiplicative functionals. By an $\varepsilon$-multiplicative functional on a Banach algebra $A$ we mean a linear functional $F$ on $A$ such that

$$
|F(a b)-F(a) F(b)| \leq \varepsilon\|a\|\|b\| \quad \text { for } a, b \in A .
$$

Such functionals play a crucial role in the investigation of small deformations of multiplication but they are also interesting in their own right. To get an example of an almost multiplicative functional, just take a multiplicative functional $G$ and any linear functional $\Delta \in A^{*}$ with sufficiently small norm, and put $F=G+\Delta$. A Banach algebra is called functionally stable if this is the only way to obtain an almost multiplicative functional; that is, if any almost multiplicative functional is close to a multiplicative one. In 1986 B. Johnson proved [14] that the Banach algebras $C(X)$, the disc algebra 
$A(\mathbb{D})$, and some other related uniform algebras are functionally stable. He also constructed a commutative radical Banach algebra which had no multiplicative functionals but had $\varepsilon$-multiplicative functionals for any $\varepsilon>0$. The first example of nonfunctionally stable uniform algebra was given by Sidney [22] in 1997. More recently the problem was investigated in [12]; however, a number of important questions remains open even for Banach algebras. For example we do not know if the algebra $H^{\infty}(\mathbb{D})$ is functionally stable - in view of the importance of the corona theorem it would be particularly interesting to know if the algebra $H^{\infty}(\mathbb{D})$ has an almost corona consisting of almost multiplicative functionals far from the maximal ideal space of $H^{\infty}(\mathbb{D})$. The concept of almost multiplicative functionals can be easily extended to topological algebras along the same lines as the extension of deformations of multiplication. There is an abundance of interesting natural open problems here, for example: Which topological algebras are functionally stable? Is multiplicative stability of an $m$-convex algebra $\left(A, \cdot, p_{\alpha}\right)$ equivalent to functional stability of the completions of all quotient algebras $A / \operatorname{ker} p_{\alpha}$ ? etc.

To go even further one can ask about almost multiplicative maps between two topological algebras or about continuous/analytic structures on the family of all deformations of an algebra; partial results are again available only for very special Banach algebras ([14], [5], [21]).

\section{References}

[1] E. Beckenstein, L. Narici, and C. Suffel, Topological Algebras, North-Holland, 1977.

[2] M. Cambern and K. Jarosz, Ultraproducts, $\varepsilon$-multipliers, and isomorphisms, Proc. Amer. Math. Soc. 105 (1989), 929-937.

[3] M. Fragoulopoulou, Q-algebras: how close are they to Banach algebras, in: General topological algebras (Tartu, 1999), Math. Stud. (Tartu) 1, Est. Math. Soc., 2001, $125-134$.

[4] S. Heinrich, Ultraproducts in Banach space theory, J. Reine Angew. Math. 313 (1980), 72-104.

[5] K. Jarosz, Perturbations of Banach Algebras, Lecture Notes in Math. 1120, Springer, 1985.

[6] -, Perturbations of uniform algebras, II, J. London Math. Soc. 31 (1985), 555-560.

[7] - Small isomorphisms between operator algebras, Proc. Edinburgh Math. Soc. 28 (1985), 121-131.

[8] - $H^{\infty}(\mathbf{D})$ is stable, J. London Math. Soc. 37 (1988), 490-498.

[9] - Perturbations of function algebras, in: NATO Adv. Inst. Sci. Ser. C, Kluwer, 1988, 557-563.

[10] - Small perturbations of algebras of analytic functions on polydiscs, in: K. Jarosz (ed.), Function Spaces, Dekker, 1991, 223-240.

[11] -, Ultraproducts and small bound perturbations, Pacific J. Math. 148 (1991), 81-88.

[12] —, Almost multiplicative functionals, Studia Math. 124 (1996), 37-58.

[13] B. E. Johnson, Perturbations of Banach algebras, Proc. London Math. Soc. 35 (1977), 439-458. 
[14] B. E. Johnson, Approximately multiplicative functionals, J. London Math. Soc. 35 (1986), 489-510.

[15] R. Rochberg, Almost isometries of Banach spaces and moduli of planar domains, Pacific J. Math. 49 (1973), 445-466.

[16] - , Almost isometries of Banach spaces and moduli of Riemann surfaces, Duke Math. J. 40 (1973), 41-52.

[17] - Algebras of analytic functions on degenerating Riemann surfaces, Bull. Amer. Math. Soc. 81 (1975), 202-204.

[18] —, Almost isometries of Banach spaces and moduli of Riemann surfaces, II, Duke Math. J. 42 (1975), 167-182.

[19] —, Deformation of uniform algebras, Proc. London Math. Soc. 39 (1979), 93-118.

[20] - , The disc algebra is rigid, ibid., 119-130.

[21] - Deformation of uniform algebras on Riemann surfaces, Pacific J. Math. 121 (1985), 135-181.

[22] S. J. Sidney, Are all uniform algebras AMNM?, Bull. London Math. Soc. 29 (1997), 327-330.

[23] W. Żelazko, Extending seminorms in locally pseudoconvex algebras, in: Functional Analysis and Operator Theory (New Delhi, 1990), Lecture Notes in Math. 1511, Springer, 1992, 215-223.

University of Tartu

50409 Tartu, Estonia

E-mail: abel@math.ut.ee
Southern Illinois University Edwardsville, IL 62026, U.S.A.

E-mail: kjarosz@siue.edu Web: http://www.siue.edu/ ${ }^{\sim}$ kjarosz/

Received September 7, 2001

Revised version February 3, 2003 